\title{
From the principle of least action to the conservation of quantum information in chemistry. Can one generalize the periodic table?
}

\author{
Vasil Penchev, vasildinev@gmail.com \\ Bulgarian Academy of Sciences: Institute of Philosophy and Sociology: \\ Dept. of Logical Systems and Models
}

\begin{abstract}
In fact, the first law of conservation (that of mass) was found in chemistry and generalized to the conservation of energy in physics by means of Einstein's famous " $E=m c^{2}$ ". Energy conservation is implied by the principle of least action from a variational viewpoint as in Emmy Noether's theorems (1918): any chemical change in a conservative (i.e. "closed") system can be accomplished only in the way conserving its total energy. Bohr's innovation to found Mendeleev's periodic table by quantum mechanics implies a certain generalization referring to the quantum leaps as if accomplished in all possible trajectories (according to Feynman's interpretation) and therefore generalizing the principle of least action and needing a certain generalization of energy conservation as to any quantum change. The transition from the first to the second theorem of Emmy Noether represents well the necessary generalization: its chemical meaning is the generalization of any chemical reaction to be accomplished as if any possible course of time rather than in the standard evenly running time (and equivalent to energy conservation according to the first theorem).

The problem: If any quantum change is accomplished in all possible "variations (i.e. "violations) of energy conservation" (by different probabilities), what (if any) is conserved?

An answer: quantum information is what is conserved. Indeed, it can be particularly defined as the counterpart (e.g. in the sense of Emmy Noether's theorems) to the physical quantity of action (e.g. as energy is the counterpart of time in them). It is valid in any course of time rather than in the evenly running one. That generalization implies a generalization of the periodic table including any continuous and smooth transformation between two chemical elements.
\end{abstract}

Key words: conservation, Emmy Noether's theorems of conservation, quantum information, periodic table, quantum chemistry 


\section{A "Gedankenexperiment" in Einstein's manner as an introduction}

A thought experiment is a possible generalization of that of Einstein (1956: $\S 22)^{1}$ :

Let us imagine an observer of a certain chemical reaction occuring in an arbitrary reference frame: for example, an astrochemist observing from the earth a chemical reaction in a very remote celestial body in the universe by means of the spectral lines, i.e. the process of their transformation from belonging to a set of chemical substances to that of another.

One may express the same from the viewpoint of quantum mechanics as the transformation of a wave function of initial chemical substances ${ }^{2}$ to that of another. Energy conservation, as a universal law of all chemical reactions in the universe, would imply for the operator transforming the initial wave function into the final one to be a Hermitian (self-adjoint) operator.

However, the earthly astrochemist would observe a quite different operator linked to the same chemical reaction if it occurred on the earth. That operator would not be Hermitian in general and thus, it would suggest a certain violation of energy conservation in the course of chemical reaction. Anyway, he or she will be able to explain the observed difference very easily referring to the curving and distortion added by the deviation of the real geodetic line, in which the spectral lines reach the earth, to the straight line, according to Einstein's theory of general relativity (Einstein 1916; 1918). Thus, a one-to-one mapping of all arbitrary geodetic lines in the pseudo-Riemannian space of the universe and all possible deviations of the operators of a certain chemical reaction, observed anywhere in the universe by the earthly astrochemist, to the Hermitian operator of the same reaction observed on the earth can be constructed in principle.

Furthermore, the astrochemist accomplishes another set of experiments about the course (i.e. the operator) of the same chemical reaction rigorously restricted to be on the earth, however under the condition of a quite different set of variables: all possible entanglements of the initial chemical substances resulting into a set of subspaces non orthogonal both to each other and to that of final compound of the reaction, in general.

One can represent equivalently any given entanglement of the wave functions of the initial, " $n$ " chemical substances, " $\Psi_{\mathbf{n}}$ ", into the Hilbert space, " $\mathfrak{Y}$ ", to which of the wave function of the final chemical compound " $\Psi \mathbf{f}$ " as a single wave function, " $\boldsymbol{\psi}$ ", by means of the following procedure determining unambiguously the members of $\boldsymbol{\Psi}_{\mathbf{f}}, " \mathbf{C}_{\mathbf{k}} \mathbf{e}^{\mathbf{i}[\mathbf{k}+\boldsymbol{\varphi}(\mathbf{k})] "}$

$1 . \mathfrak{H}$ is divided into $\mathbf{n}$ subspaces, $\mathfrak{H}_{\mathbf{n}}$, non-orthogonal to each other in general, corresponding to the $\mathbf{n}$ initial chemical substances and their $\mathbf{n}$ wave functions.

2. As far as the initial chemical substances are entangled, the intersections of any two subspaces $\mathfrak{H}_{\mathrm{j}}$ and $\mathfrak{H}_{\text {I }}$ will not be empty in general.

3. The member $\mathbf{C}_{\mathbf{k}} \mathbf{e}^{\mathbf{i}[\mathbf{k}+\boldsymbol{\varphi}(\mathbf{k})]}$ is determined unambiguously as the vector sum of the corresponding $\mathbf{k}^{\text {-th }}$ members of all wave functions of the initial chemical substances.

\footnotetext{
${ }^{1}$ The paper of Norton (1984) reveals the importance of that thought experiment for creating the theory of general relativity by Albert Einstein.

${ }^{2}$ As the initial chemical substances are separated from each other, their wave functions are orthogonal to each other and may be considered in orthogonal subspaces of a single and shared Hilbert space utilized by quantum mechanics, and thus, as a single wave function.
} 
The operator, $\mathbf{0}$, defined as $\boldsymbol{\Psi} \rightarrow \boldsymbol{\Psi}_{\mathrm{f}}$ is not Hermitian in general. That procedure generates a mapping of the set of all possible entanglements of the initial chemical substances of the investigated chemical reaction into the set of all operators transforming $\mathfrak{H}$ into itself.

So, one will obtain the results of two class of experiments. The former class, " $\mathcal{F}$ ", consists of all results of the observations of a given chemical reaction in all points of the universe, accomplished by the earthly astrochemist. The latter class, " $\mathcal{L}$ ", consists of the set of all chemical reactions on the earth and derived by the same chemical reaction as in the former case under the condition to be considered all possible entanglements of the initial chemical substances of the same reaction. Both classes can be mapped into the set of all operators of the Hilbert space into itself supposedly one-to-one. If that is the case, a one-to-one mapping between $\mathcal{F}$ and $\mathcal{L}$ exists necessarily.

Following the narrative structure of Einstein's Gedankenexperiment, one can visualize the hypothesis of the equivalency of $\mathcal{F}$ and $\mathcal{L}$ on the base of the supposed one-to-one mapping between them in different ways, e.g. so:

The results of both classes experiments turn out to be mixed because of some extraordinary circumstances: e.g. a hacker attack upon the astrochemist's computer. Thus, he or she need divide them into the original two classes before the attack. The hypothesis implies: this will be impossible for any result will be doubled by the same counterpart. Or: the astrochemist mixes the results on purpose and suggests to any colleague to divide them into the initial classes: the colleague will be not able to do it unambiguously for the same reason.

In fact, that thought experiment repeats the logical and even narrative structure of Einstein's Gedankenexperiment about an elevator whether accelerated or falling in gravitational field. This is not occasional: it will be used also further.

\section{The missing link}

That missing link is the correspondence of a geodetic line (or trajectory) in the pseudoRiemannian space of general relativity and an element ("wave function") of the separable complex Hilbert space utilized by quantum mechanics, in the following sense:

Any mathematical proof consists of a series (often enormous, tiring, and boring) of ruttine logical and mathematical operations and one or a few "insights" linking creatively and rigorously remote enough mathematical structures or certain elements of them, which might be called "missing links":

That "missing link" in the proof of the hypothesis about the equivalency of $\mathcal{F}$ and $\mathcal{L}$ is the above correspondence.

As to the philosophical and fundamental essence of the problem, one may omit that ruttine series and explicate only the missing link: what we will do as follows:

First, one can ground why namely that is the missing link:

The chemical reaction observed from an immovable reference frame anywhere in the universe is described by the same Hermitian operator, $\mathbf{O}_{\mathbf{H}}=\boldsymbol{\Psi}_{\mathbf{0}} \rightarrow \boldsymbol{\Psi}_{\mathrm{f}}$ (where $\boldsymbol{\Psi}_{\mathbf{0}}$ means the wave function 
of the unentangled initial chemical substances of the reaction at issue) in virtue of the universality of the laws as physical as chemical anywhere in the universe.

However, the earthly astrochemist observes it as $\mathbf{0}=\mathscr{R}\left(\mathbf{0}_{\mathbf{H}}\right)=\mathscr{R}\left(\boldsymbol{\Psi}_{\mathbf{0}} \longrightarrow \boldsymbol{\Psi}_{\mathbf{f}}\right)$, and $\mathscr{R}\left(\boldsymbol{\Psi}_{\mathbf{0}} \longrightarrow \boldsymbol{\Psi}_{\mathbf{f}}\right)$ $=\mathscr{R}\left(\boldsymbol{\Psi}_{\mathbf{0}}\right) \rightarrow \mathscr{R}\left(\boldsymbol{\Psi}_{\mathbf{f}}\right)$, under the condition the time of the reaction in any immovable reference frame in the universe to be zero to the time for the spectral lines of the reaction to reach the earth propagating by light speed and following the corresponding geodetic line, " $\mathscr{R} "$, in the space-time of the universe according to general relativity: so, $\mathscr{R}=\mathbf{0}_{\mathbf{H}} \longrightarrow \mathbf{0}$.

On the other hand, one can suggest exactly one single counterpart of $\mathscr{R}$, " $\mathcal{G}$ ", in the latter class of experiments $\mathcal{L}$ such that: as $\mathbf{0}=\mathcal{E}\left(\mathbf{O}_{\mathbf{H}}\right)=\mathcal{E}\left(\boldsymbol{\Psi}_{\mathbf{0}}\right) \rightarrow \boldsymbol{\Psi}_{\mathbf{f}}$, so:

$$
\begin{aligned}
& \mathcal{E}\left(\boldsymbol{\Psi}_{\mathbf{0}}\right)=\mathscr{R}\left(\boldsymbol{\Psi}_{\mathbf{0}}\right) \\
& \mathscr{R}\left(\boldsymbol{\Psi}_{\mathbf{f}}\right)=\boldsymbol{\Psi}_{\mathbf{f}} .
\end{aligned}
$$

In other words, this is a system of two equations with two unknowns, $\mathcal{E}$ and $\mathscr{R}$, correspondingly operators in Hilbert space and in pseudo-Riemannian space, and thus having one single solution, which is equivalent to a one-to-one mapping of the Hilbert space of quantum mechanics, and pseudo-Riemannian space of general relativity if that system of equation is resolved as to all points in the universe and all possible entanglements of the initial chemical substances of the etalon reaction.

Returning backwards, if one constructs a one-to-one mapping of that Hilbert space , " $\mathfrak{Y}$ ", and

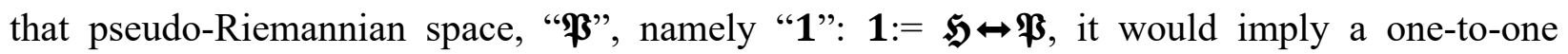
mapping of the results of the two classes of experiments, $\mathcal{F}$ and $\mathcal{L}$. Thus, this is the ultimate consideration about why " $\mathbf{1}$ " is the missing link. Furthermore, " $\mathbf{1}$ " should have a clear and convincing physical interpretation (meaning) as far as both " $\mathfrak{H}$ " and " $\mathfrak{P}$ " possess those, correspondingly in quantum mechanics and general relativity.

Secondly, one can suggest an unambiguous transform between a geodetic line ("trajectory") in the latter and a point ("wave function") in the former.

A few main mismatches stand out and sting the eye immediately: " $\mathfrak{H}$ " is (1) infinitely

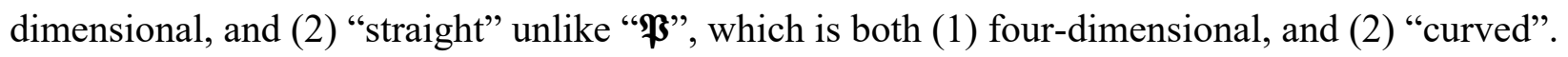
One can add a few statements more: (3) the measure in " $\mathfrak{H}$ " is normal unlike the pseudo-measure of " $\mathfrak{P}$ " (shared with, or inherited from the Minkowski space of special relativity); and (4): quantum mechanics utilized " $\mathfrak{H}$ " uses it to describe discrete ("quantum") physical changes unlike general relativity describing continuous and even "smooth" (in mathematical sense) changes by means of " $\mathfrak{P}$ " though exceptions as singularities are admissible; and (5): if one consider both as vector spaces, the pseudo-Riemannian space of general relativity is real unlike the Hilbert space of quantum mechanics, which is complex. This means: the former vector space is defined on the field of real numbers, the latter, on that of complex numbers.

The searched " $\mathbf{1}$ " is necessary to unify the above mismatches from its generalizing viewpoint. Still one, very important, physical and philosophical rather than mathematical restriction is to be added: the searched " $\mathbf{1}$ " need transform unambiguously a certain trajectory of general relativity

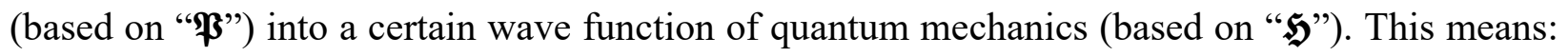
(1) a trajectory in general relativity is not only a geodetic line in pseudo-Riemannian space, but a 
meaningful series of experimental data referring to the theory of general relativity; (2) a wave function in quantum mechanics is not only a point in Hilbert space, but a meaningful series of experimental dates referring to the theory of quantum mechanics; and (3): " 1 " need be interpretable physically meaningfully, i.e. it is necessary to transform the former series of data into the latter one unambiguously.

Properly mathematically, the problem is formulated so: its solutions, " $\mathbf{1}$ ", constitutes the set of all mappings transforming one-to-one the set of all geodetic lines of a given pseudo-Riemannian space (that utilized by general relativity) into the set of all points of a certain Hilbert space (utilized by quantum mechanics). It seems not to be too difficult as far as those "geodetic lines" and "points" belong to the same set of functions therefore determining additionally the solution as the mappings of one subset of that set to another of it.

Further, one need choose from " $\mathbf{1}$ " at least one element satisfying the above physical and mathematical restriction.

Following the sketched already approach of "missing link", one is sufficient to demonstrate a physically meaningful element of " 1 " furthermore unifying and thus "matching" the above, (1) (5) mismatches between " $\mathfrak{H}$ " and " $\mathfrak{P}$ ":

One can consider any trajectory in the space-time of general relativity as an instantaneous and simultaneous "leap", and also vice versa: any leap described by a wave function in quantum mechanics as a continuous, even smooth "trajectory" occurring successively, "point by point" rather than simultaneously. Mathematically, the Fourier transform and its reverse transform are able to express that transition from a trajectory to a leap, and then: vice versa. The former replaces the fundamental variable of time, "t', in space-time by the fundamental variable of energy, "E", (literally that of frequency, "f') unambiguously for " $\mathbf{E}=\mathrm{h} / \mathbf{t}$ " involving the fundamental Planck constant, "h" (respectively, the latter: "t$=\mathrm{h} / \mathbf{E}$ ").

In terms of the theory of general relativity, that transform can be described conceptually by generalizing the notion of reference frame as to "discrete" ones: each of them can be defined unambiguously by a certain "leap" in space-time to a given reference frame; or alternatively and equivalently: as the class of reference frames being "out of the universe" (and thus, reachable only by a leap).

In terms of the theory of quantum mechanics, the reverse transform can be described conceptually by the following construction actualizing the wave function in the sense of the manyworlds interpretation suggested by Hugh Everett III (1957; DeWitt, Wheeler 1968):

One consider the same point (quantity) successively in a well-ordered series of "worlds", each of which is infinitely close to the next one of the series. Mathematically, this means: one restores (1) a probability distribution from its characteristic function by means of the reverse Fourier transform; and (2): the reverse function of the probability distribution, i.e. therefore the value of quantity (trajectory) "world" (a value of probability) by "world" (another and infinitely close value of probability).

However, the terms of the theory of the so-called Standard model suggests the most generalizing viewpoint to the interpretation of the physically meaningful one-to-one mapping 
between the set of trajectories and the set of wave functions as above. Furthermore, they are linked to Emmy Noether's theorems (Noether 1918)

The theory of the Standard model introduces both "global space" and "local space", in which any quantum change occurs:

The global space is shared with classical physics and thus, with the macroscopic "apparatus" recording by its readings the state of the investigated quantum entity. Its symmetries are "global" and correspond to the conservation laws of classical physics (including special relativity).

The local space is irrelevant to classical physics: it refers only to quantum mechanics and may be visualized by two different ways contradictory to each other at first glance, but not in fact:

On the one hand, it is as if "hidden" within any point of the trajectory in the global space. On the other hand, it represents the global space as a whole as if observed "from outside": from "a point out of the universe".

The two visualizations turn out to be reconciled to each other if one utilizes the cyclic viewpoint from the "biggest" entity (the universe) to the "smallest" one (a quantum) in Nicholas of Cusa's manner.

The symmetries of that local space imply corresponding local, quantum laws of conservation, according to the second theorem of Emmy Noether (1918), unlike the analogical correspondence in the global space being the subject of the first one (Noether 1918).

In the so-sketched terms of the Standard model, the one-to-one mapping at issue means the the pairs of counterparts, the one of which belongs to the global space, the other, to the local one. Furthermore, still one and generalizing invariance (respectively symmetry and conservation) can be outlined: that between global space and local space in the sense of the Standard model. It will be discussed in the next section.

At last, one can display that the mismatches "(1) - (5)" are fitted to each other by means of that mapping of a trajectory into a wave function. Indeed:

(1): “ $\mathfrak{H}$ " is infinitely dimensional unlike " $\mathfrak{P}$ ", which is four-dimensional: however, we map one-to-one subsets of " $\mathfrak{P}$ " ("trajectories") and elements of " $\mathfrak{Y}$ " ("wave functions").

(2) and (5): “ $\mathfrak{G}$ " "straight" unlike " $\mathfrak{P}$ ", which is "curved". However the former is complex unlike the latter, which is real. A "straight" and complex vector space can be equivalent to a "curved" and real one as far as the real and imaginary part of a complex vector are able to represent unambiguously two independent and thus different vectors: the one of which is covariant, the other, contravariant.

(3) The pseudo-measure of " $\mathfrak{P}$ " representing mathematically the physical restriction of light speed corresponds to the limitation of all wave functions to be characteristic function of probability distributions and thus restricted to a unit though the measure of " $\mathfrak{H}$ " is normal rather than a pseudo one

${ }^{3}$ The book of Kosmann-Schwarzbach (2011) contains an overview of the contemporary viewpoint to the theorems. 
(4) The Fourier transform (respectively its reverse) maps the "beginning" of a smooth function into the converging "end" of a discrete series of the coefficients of the complex vector equivalent to a wave function (respectively: vice versa).

Fitting all mismatches between " $\mathfrak{P}$ " and “ $\mathfrak{H}$ ", one can assure that the same mathematical structure is meant though described by two different ways, even absolutely irrelevant to each other at first glance, however equivalent in fact.

\section{The missing link in terms of quantum information}

At the end of the last century, quantum mechanics was reformulated thoroughly in terms of a generalized kind of information: quantum information ${ }^{4}$. As far as quantum mechanics has underlain chemistry since the first quarter of $20^{\text {th }}$ century, quantum information also should be able to ground chemistry ${ }^{5}$.

If the quantity of classical information (respectively, entropy) is measured by "bits", the quantity of quantum information is measured in "quantum bits" or "qubits". A bit is equivalent to an elementary choice between two equiprobable alternatives. A qubit being a generalization of "bit" is equivalent to the choice between an infinite set of alternatives (Penchev 2016).

Furthermore, a qubit is defined in quantum mechanics as the normed superposition of two orthogonal subspaces (such as two "axes", most often) of the separable complex Hilbert space. By that formulation, as any wave function as any entanglement can be represented tautologically as a series of qubits therefore involving a unification of Hermitian and non-Hermitian operators from the viewpoint of quantum information.

Thus, the concept of quantum information allows of generalizing energy conservation to the case of entanglement, on the one hand, and to the case of general relativity (also, a non-unitarian theory), on the other nand. We need therefore "quantum information" as far as the initial thought experiment and further consideration discuss right the equivalency of experiments on entanglement and observations on a chemical reaction occuring in a remote enough object in the space-time of general relativity.

Indeed, the missing link in question can be represented very easily and simply in terms of quantum information by the following construction:

Any qubit is mathematically isomorphic and thus equivalent to a unit ball, two points of which are chosen: the one within the ball, the other on its surface. That unit ball with chosen two points as above is equivalent to two three-dimensional vectors orthogonal to each other and both less than

\footnotetext{
${ }^{4}$ The book of Timpson (2013, as well as: Penchev 2009) contains an overview of that reformulation.

5 A brief overview about the application of quantum information in chemistry is available in: Penchev, V. (2016). Problem of the direct quantum-information transformation of chemical substance (§ 1) available at the following web address $(28.05 .2019)$ or by the title in a google search: https://www.researchgate.net/publication/305330641 Problem of the direct quantuminformation transformation of chemical substance Their results are not necessary for the statements in the present paper.

${ }^{6}$ The concept of qubit can be visualized even in an absolutely rigorous meaning (Penchev 2015) by Einstein's metaphor for "God's dice" (which he rejected to be relevant to physics until the end of his life).
} 
a unit. Further, the one of them can be interpreted as the covariant vector and the contravariant vector in a certain point of pseudo-Riemannian point.

Thus, any infinite series of qubits has two equivalent interpretations: as the separable complex Hilbert space of quantum mechanics as the pseudo-Riemannian space of general relativity: representing right the "missing link" in terms of quantum information

\section{The conservation of quantum information by Emmy Noether's theorems (1918)}

One can suggest the following consideration to introduce the conservation of the quantity of quantum information in the sense of her theorems:

(a) What is meant as a universal physical quantity in the theorems at issue is action and only action. So, they privilege action as the most fundamental, both physical and chemical quantity, by which all others are underlain. That privileging is not invented by them, but it is rather extracted by the development of mechanics (including quantum mechanics), where the principle of least action is one of the most fundamental and heuristic ones and formulated explicitly in various and generalized forms a long time ago.

(b) They discuss any possible division of the action within any physical system into two parts independent of each other: the one is "what is changed"; the other one and its counterpart is "what is conserved".

(c) So, they state implicitly (or as their condition) that change and conservation in any physical or chemical system can be distinguished from each other disjunctively and furthermore described quantitatively independently of each other always. Action is that fundamental physical quantity able to unify those two metaphysical categories (namely change and conservation) as to all the physical.

(d) Only on that base sketched in "a-c", the explicit statements of both theorems can be elucidated in a generalized way: they reveal the link of the law of change by means of its " $n$ " variables or "parameters" and the law of conservation by means of its " $n$ " differential equations to be extremely simple. Their number is the same, "n". Particularly, the first theorem investigates the "degenerated" case where all variables of change are constants, or properly "parameters", and the corresponding differential equations of conservation are in turn "degenerated" to the " $n$ " times repeated tautology "the differential of any constant is zero"'

(e) If one involves the concept of 'symmetry' as the "constancy of change", the usual focus of interpretation centred on the first theorem may be restored secondarily in our viewpoint as the coincidence of the "constancy of change" with the "constancy of conservation".

(f) Meaning our intention further to involve '(quantum) information' as the counterpart of the physical action itself, still one similarity needs a certain formulation expressively. The formal

\footnotetext{
7 One may notice, that the solution of the system of "differential equations" in that degenerate case can be directly interpreted as the variables of change, both being " $n$ " constants. One may admit that the interpretation of the solution of the equations as the variables of change might hold in the case of the second theorem. However, it does not state this, but something much weaker, which is a trivial corollary from the much stronger coincidence of the variables of change and the variables-solution of the system of differential equations of conservation.
} 
structure of the disjunctive division of the action into two correlative counterparts of change and conservation correspondingly possesses the formal structure of a $\mathrm{bit}^{8}$ of information.

Resuming, Noether's theorem determine the links between the conserved quantities, e.g. energy, and the changeable, e.g. time, in any physical system. The product of the former and the latter has always the physical dimension of action (what the dimension of the fundamental Planck constant is, by the way) and thus, it can be interpreted as the physical quantity of action. One can consider the particular case where what is changed is physical action. If what is changed is physical action, the theorems of Emmy Noether imply that what is conserved should be dimensionless physically. Quantum information being namely dimensionless physically seems to be an admissible applicant for the counterpart of action. Our thesis is:

Quantum information is conserved, being the counterpart of the changeable action.

Two examples may elucidate the meaning of the first Noether theorem to both action as what is changed and quantum information as what is conserved:

(1) Global symmetry of action in quantum mechanics. If the functional of action is identical to the action itself as far as the interpretation of the action itself as what is changed requires in virtue of the first Noether theorem, this implies the symmetry of Hilbert space utilized in quantum mechanics and its dual counterpart identical to the former. That Hilbert space can be interpreted as the free variable of quantum information and any wave function as a certain value ${ }^{9}$ of it. Consequently, that global symmetry of action implies the conservation of quantum information as the Hilbert space of quantum mechanics is its universal mathematical formalism.

(2) Global symmetry of action in general relativity. A main obstacle fortunately soluble exists for the approach to the global symmetry of action in quantum mechanics to be repeated as to general relativity: its pseudo-Riemannian space is "curved", and thus the corresponding dual counterparts (the covariant one and the contravariant one) are different. Its pseudo-metrics and finite dimensionality are not any trouble, for the imaginary (physically, subluminal) domain of Minkowski space being also pseudo-metrical and four-dimensional admits an isomorphism to the separable complex Hilbert space of quantum mechanics (Penchev 2013) under the additional condition of the axiom of choice ${ }^{10}$. So, the problem would be resolved if one builds a physically meaningful isomorphism between the real (physically, superluminal) domain and the imaginary (subluminal physically) domain of pseudo-Riemannian space (Penchev 2013) for a very simple symmetry exists between the real and imaginary domain of Minkowski space. Action is an internal quantity to those spaces physically interpreted ${ }^{11}$, and consequently that series of isomorphisms

\footnotetext{
${ }^{8}$ It can be defined as the disjunctive choice between two equally probable alternatives, which may be "change" and "conservation".

${ }^{9}$ The variable of quantum information can be visualized as a quantum machine of Turing, i.e. as an infinite series of qubits; correspondingly, "free variable", as "empty" sells without any recording in each of them, and "wave function" as the well-ordered set of certain records in each of them.

${ }^{10}$ Involving the axiom of choice is justified intuitively as the "set-theory quantization" therefore referring to Skolem's "paradox" (or said better, "Skolem's argument”: Skolem 1923).

${ }^{11}$ Action is defined as a variable of both dual counterparts within each space in question.
} 
conserves any symmetry of action implying a global symmetry of action in general relativity very similar and even identical in essence to the analogical symmetry in quantum mechanics.

If the change of action is uniform in time, energy is conserved. However, the conservation of quantum information is more universally than energy conservation for if the action is changed (i.e. not only uniformly), its counterpart of quantum information is conserved. Energy conservation implies quantum-information conservation, but not vice versa.

One existing and exceptionally elaborated example for that generalized approach to conservation is general relativity. Energy may be transformed in momentum in it immediately because of the "curvature" of space-time in it according to its most fundamental equation: the Einstein field equation.

The above consideration involves the conservation of quantum information only in the terms of the first theorem, i.e. as a global conservation as those in classical physics. However, the concept of quantum information just as the "missing link" bridges and thus unifies both global and local spaces in terms of the Standard model, both local and global symmetries (respectively, conservation laws) in terms of Noether's theorems.

One can consider the conservation of quantum information also "locally" e.g. by means of Feynman's approach and visualization "in many paths":

A certain probability corresponds to each "axis" of the Hilbert space of quantum mechanics after Born's interpretation (Born 1926; 1927; 1954). As far as an exactly determined probability is juxtaposed, in turn, to each path of Feynman's, the latter can be interpreted as all "axes": after that, "wave function" means the corresponding quantum leap being accomplished in each one of Feynman's path "locally" and in all of them "globally".

Furthemore, one can consider the global conservation of quantum information being namely "quantum" as accomplished "locally" by each possible wave function as in a certain path of Feynman's:

If the global conservation of quantum information means the physical universality of Hilbert space of quantum mechanics as in a few paragraphs above, its local conservation means a certain wave function (interpretable as that of the universe) or a certain reference frame (interpretable as that of the "Big Bang") to be privileged as conserved and thus universal (particularly, valid in any reference frame, anywhere in the universe).

\section{To a generalization of the periodic table}

The periodic table we know is grounded to two implicit premisses: (1) referring to an immovable reference frame and thus: that on the earth can be granted as an etalon; (2) referring to non-entangled states of chemical substances.

The present paper demonstrated already above, that both premisses should be unified into a single one. However, a very important difference between (1) and (2) is kept: (1) refers to "images", and it is not "real" as far as the observed course of a remote enough chemical reaction can influence no way to any chemical reaction occuring on the earth. On the contrary, (2) means the real change of chemical properties: entangled chemical substances can be involved in chemical 
reaction as such different from their non-entangled counterparts and possibly equivalent to different non-entangled substances.

Particularly or symbolically, this implies a certain generalization of the periodic table including all possible entangled states along with the non-entangled states of the "classical" periodic table. The following simplification is convenient to be utilized: any state of entangled chemical substances can be considered equivalently as the same chemical reaction as to their corresponding non-entangled counterparts excluding one single substance among the initial one, which participates by an equivalently entangled state and different from its real entangled state in the real chemical reaction of all really entangled initial substances of that reaction.

In other words, the entanglement of a system of initial chemical substances, which are going to interact immediately, can be represented by a corresponding entangled state only of a single of them.

Particularly, that simplification justifies the periodic table to be generalized as to the new chemistry of entangled states.

Indeed, the simplification can be proved as a immediate corollary:

The statement is sufficient to be demonstrated as to a single "axis" of Hilbert space for two entangled substances and confirmed twice by mathematical induction as to all "axes" as to an arbitrary number of entangled substances.

As to a single axis for only two substances:

\section{$\left\{\mathbf{C}_{1} \exp \left[\mathrm{i}\left(\mathbf{n} \boldsymbol{\omega}+\boldsymbol{\varphi}_{1}\right]\right\}\left\{\mathbf{C}_{2} \exp \left[\mathrm{i}\left(\mathbf{n} \boldsymbol{\omega}+\boldsymbol{\varphi}_{2}\right]\right\}=\left\{\mathbf{C}_{1} \exp [\mathrm{i}(\mathbf{n} \boldsymbol{\omega})]\right\}\left\{\mathbf{C}_{\mathbf{1}} \cdot \mathbf{C}_{2} \cdot \exp \left(\mathrm{i}_{1}\right)\right\}\left\{\exp \left[\mathrm{i}\left(\mathbf{n} \boldsymbol{\omega}+\boldsymbol{\varphi}_{2}\right]\right\}\right.\right.\right.$}

In other words, substituting $\mathbf{C}_{\mathbf{2}}$ with $\mathbf{C}_{2 \text { new }}=\mathbf{C}_{\mathbf{1}} \cdot \mathbf{C}_{\mathbf{2}} \cdot \exp \left(i \boldsymbol{\varphi}_{1}\right)$, one can represent the whole entanglement by the entanglement only of the one single substance in relation to the Hilbert space of the compound, which is the result of the chemical reaction.

The periodic table can be generalized including the additional variable of an arbitrary (nonHermitian in general) operator in Hilbert space acting upon the wave function of each element of the periodic table.

Still one simplification is possible. One can consider the action of that arbitrary operator upon the wave function of a single element, e.g. hydrogen being added really or formally to any chemical reaction either as an interacting substance or as a non-interacting substance. Thus, the generalization of the periodic table can be represented equivalently adding only all possible entangled states of hydrogen.

\section{The idea in the mass culture (sci-fi)}

If one might generate a directed ray of entanglement, the ray would change the properties of any chemical compound at an arbitrary distance as those of a certain other one, according to the variable of entanglement.

That idea underlies "The Trigger", a sci-fi novel by Arthur C. Clarke and Michael P. KubeMcDowell (1999): 
A collective of scientists work on a device able to generate a directed ray of gravitation, a gravitational analog of laser, but reveals occasionally that it changes chemical properties in certain cases. One of the characters elaborates a new theory underlying all chemical substances by information.

\section{Instead of a conclusion: the generalization of Einstein's Gedankenexperiment about an elevator}

The consideration of Einstein demonstrates that gravitational field is equivalent to force field just as gravitational mass is equal to inertial mass always. It underlies his theory of general relativity.

One can generalizes it further e.g. as in the present paper. That thought experiment shows that a wave function of any chemical substance or the operator of any chemical reaction anywhere in space and observed from the earth is indistinguishable from a certain entanglement of the same wave function or correspondingly, from the corresponding non-Hermitian operator in general. Its base is the analogical equality of classical information and quantum information.

Anyway, the physical action of classical information is absolutely different from that of quantum information. The former is neither equivalent to any physical action nor able to cause any physical change. On the contrary, the latter is equivalent to a certain value of the quantity of action by means of the fundamental Planck constant and it is able to cause a corresponding physical change by entanglement.

A possible way to be reconciled that quantitative equality of classical and quantum information, on the one hand, and their qualitative difference as to physical action, on the other hand, is the following:

The magnitude of classical information compared with that of quantum information is infinitely small. Thus, the physical action caused by it is infinitely small and unobservable experimentally in principle. Indeed, the unit of classical information is measured by the unit of a "bit", the choice between two equally probable alternatives. The unit of a qubit for quantum information is equivalent to the choice among an infinite set of alternatives. Consequently, any finite quantity of quantum information is infinitely big to any finite quantity of classical information. The simultaneous consideration of both classical information and quantum information needs an appropriate mathematical formalism such as the non-classical analysis of Robinson (1966).

The signal carrying the classical information of a certain chemical reaction occurring remotely is deformed in its movement in a certain geodetic line in space-time according to the very well confirmed theory of general relativity. Its deformation is smooth, changing a finite amount of classical information between any two infinitely close points of the trajectory. The deformation as to the sum effect observed on the earth is infinitely big in units of classical information, and thus, it can be expressed as a finite amount of quantum information equivalent to a certain entanglement applied to the same chemical reaction taking place on the earth.

Entanglement being equivalent to the integral deformation is able to change chemical properties. This implies a possible generalization of the periodic table. 


\section{References:}

Born, M. (1926) “Zur Quantenmechanik der Stoßvorgänge,” Zeitschrift für Physik 37 (12): $863-867$.

Born, M. (1927) "Physical aspects of quantum mechanics," Nature 119 (2992): 354-357.

Born, M. (1954). The statistical interpretation of quantum mechanics (Max Born - Nobel Lecture. NobelPrize.org. Nobel Media AB 2018. Thu. 6 Sep 2018.

$<$ https://www.nobelprize.org/prizes/physics/1954/born/lecture/>)

DeWitt, B, \& Wheeler, J. (eds.) (1967) The Everett-Wheeler Interpretation of Quantum Mechanics. Battelle Rencontres: 1967 Lectures in Mathematics and Physics. New York: W. A. Benjamin, 1968.

Einstein, A. (1916). Die Grundlage der allgemeinen Relativitätstheorie. Annalen der Physik 49 (7): 769-822.

Einstein, A. (1918). Prinzipielles zur allgemeinen Relativitätstheorie. Annalen der Physik 55 (4): 241-244.

Einstein, A. (1956). Über die spezielle und die allgemeine Relativitätstheorie. Berlin, Heidelberg, Springer, 2009.

Everett III, H. (1957). “„Relative state” Formulation of Quantum Mechanics,” Reviews of Modern Physics 29 (3): 454-462.

Kosmann-Schwarzbach, Y. (2011). The Noether Theorems. Invariance and Conservation Laws in the Twentieth Century. New York - Dordrecht - Heidelberg - London: Springer.

Noether, E. (1918) "Invariante Variationsprobleme," Nachrichten von der Gesellschaft der Wissenschaften zu Göttingen, Mathematisch-Physikalische Klasse 1918: 235-257.

Norton, J. (1984) "How Einstein Found His Field Equations: 1912-1915," Historical Studies in the Physical Sciences, 14 (2): 253-316.

Penchev, V. (2009) Philosophy of Quantum Information. Vol. 1. Einstein and Gödel, Sofia, IPhR-BAS (in Bulgarian).

Penchev, V. (2013). "The Kochen-Specker theorem in quantum mechanics: a philosophical comment," Philosophical alternatives 22 (1), 67-77; 22 (3): 74-83.

Penchev, V. (2015) "God's Dice," Actas: VIII Conference of the Spanish Society for Logic, Methodology, and Philosophy of Sciences (J. Martínez, García-Carpintero, J. Díez, S. Oms, eds.), Barcelona, Universitat de Barcelona, 2015, pp. 297-303.

Penchev, V. (2016). "Matter as Information. Quantum Information as Matter," Nodi. Collana di Storia della filosofia 2016 (2): 127-138.

Robinson, A. (1966). Non-standard analysis, Amsterdam, North-Holland Publishing. Timpson, C. G. (2013) Quantum Information Theory and the Foundations of Quantum mechanics, Oxford, Clarendon Press.

Skolem, T. (1923) "Einige Bemerkungen zur axiomatischen Begründung der Mengenlehre," in Matematikerkongressen $i$ Helsingrofs den 4-7 Juli 1922, Den femte skandinaviska matematikerkongressen, Redogörelse. Helsinki: Akademiska-Bokhandeln, pp. 217-232. (Also in: T. Skolem. Selected works in logic (ed. E. Fenstad), Oslo, etc: Univforlaget, 1970, pp. 137-152.) 